\title{
Significative Learning Using Alpha-Beta Associative Memories
}

\author{
Catalán-Salgado Edgar Armando ${ }^{1}$, Yáñez-Márquez Cornelio ${ }^{2}$, \\ and Figueroa-Nazuno Jesus ${ }^{3}$ \\ 1 ESCOM-IPN, Av. Juan de Dios Batiz s/n, GAM, Mexico DF \\ ecatalanb05@sagitario.cic.ipn.mx \\ WWW. edgarcatalan.com \\ 2 CIC, Av. Juan de Dios Batiz s/n, GAM, Mexico DF \\ cyanez@cic.ipn.mx \\ www.alfabeta.org \\ 3 CIC, Av. Juan de Dios Batiz s/n, GAM, Mexico DF
}

\begin{abstract}
The main goal in pattern recognition is to be able to recognize interest patterns, although these patterns might be altered in some way. Associative memories is a branch in AI that obtains one generalization per class from the initial data set. The main problem is that when generalization is performed much information is lost. This is mainly due to the presence of outliers and pattern distribution in space. It is believed that one generalization is not sufficient to keep the information necessary to achieve a good performance in the recall phase. This paper shows a way to prevent information loss and make more significative learning allowing better recalling results.
\end{abstract}

\section{Introduction}

One of the main tasks in $\mathrm{AI}$ is pattern recognition. In pattern recognition the goal is to train a model to be able to recognize certain patterns of interest although these might be affected in some way. In supervised learning this training is performed by a set of patterns called the fundamental set. With this set the model obtains its generalization in the learning phase. In the recall phase the model is asked for the pattern class.

The first models of learning matrices appeared more than four decades ago 12]. Associative memories have attracted researchers due to its properties, including their efficiency, tolerance to noise and ease of implementation that facilitates the development of applications 134.

Alpha-Beta associative memories have had a noteworthy development. Since their creation [56] an algorithm that speeds up learning[7] has been developed and they (or a variant) are used in different applications 8, 9 .

In Alpha-Beta associative memories however, due to the nature of the memories, the presence of outliers affects their recall capacity; reducing it greatly. A similar problem arises when learning a great quantity of patterns in some class. This problem is called memory saturation. This paper proposes a method to 
avoid these problems and increase memory efficiency. This is achieved through the use of sub-generalizations.

Section 2 first describes the original Alpha-Beta associative memories and introduce the Alpha and Beta operators. Section 3 describes our proposal beginning with all the necessary definitions in order to build an Alpha-Beta associative committee. Learning and recall algorithms are shown in this section. Section 4 shows the experimentation and finally Section 5 presents conclusions and future work.

\section{Alpha-Beta Associative Memories}

In Alpha-Beta associative memories an input pattern of $n$ attributes is denoted by a column vector $\mathbf{x}=\left[x_{1}, x_{2}, \ldots, x_{n}\right]$ and an output pattern of $m$ attributes is denoted by a column vector $\mathbf{y}=\left[y_{1}, y_{2}, \ldots, y_{m}\right]$, where $m, n \in \mathbf{N}$.

$\left(\mathbf{x}^{\mu}, \mathbf{y}^{\mu}\right)$ is written to denote that input vector $x^{\mu}$ is associated to output vector $y^{\mu}$, with $\mu \in\{1,2, . ., p\}$ and $p$ is the number of associations. The set of associations used in the learning phase is know a priori, it is finite and called the fundamental set. The patterns belonging to the fundamental set are called fundamental patterns. The input and output patterns are represented as follows:

$$
\mathbf{x}^{\mu}=\left[\begin{array}{c}
x_{1}^{\mu} \\
x_{2}^{\mu} \\
\ldots \\
x_{n}^{\mu}
\end{array}\right] \in A^{n} \quad \mathbf{y}^{\mu}=\left[\begin{array}{c}
y_{1}^{\mu} \\
y_{2}^{\mu} \\
\ldots \\
y_{m}^{\mu}
\end{array}\right] \in A^{m}
$$

where $\mathbf{A}=\{0,1\}$. An associative memory $\mathbf{M}$ is a system that relates an input pattern $\mathbf{x}^{\mu}$ with its respective output pattern $\mathbf{y}^{\mu}$ for each $\mu \in\{1,2, \ldots, p\}$. These memories have two phases.

The first phase is called learning phase; in it the memory establishes the association between the input pattern $\mathbf{x}^{\mu}$ and output pattern $\mathbf{y}^{\mu}$. Since $\mathbf{M}$ is a matrix generated by these associations, the transposition of $\mathbf{x}^{\mu}$, denoted as $\left(\mathbf{x}^{\mu}\right)^{t}$ is used.

$$
\left(\mathbf{x}^{\mu}\right)^{t} \rightarrow \mathbf{M} \leftarrow \mathbf{y}^{\mu}
$$

An associative memory $\mathbf{M}$ is represented by a matrix with $\mathrm{ij}$-th component $m_{i j}$. If $\mathbf{x}^{\mu}=\mathbf{y}^{\mu}$ for all $\mu \in\{1,2, . ., p\}$ then $\mathbf{M}$ is auto-associative, otherwise it is hetero-associative. In the later it is possible to establish that $\mu \in\{1,2, \ldots, p\}$ for which $\mathrm{x}^{\mu} \neq \mathbf{y}^{\mu}$.

The second phase is called recall phase. In it an input pattern $\tilde{\mathbf{x}}$ is presented to the memory. This pattern may be an unknown or altered pattern, as the resulting memory gives an output pattern $\mathbf{y}$ :

$$
(\tilde{\mathbf{x}})^{t} \rightarrow \mathbf{M} \rightarrow \mathbf{y}
$$


If the input pattern $\tilde{\mathbf{x}}=\mathbf{x}^{\mu}$ for some $\mu \in\{1,2, . ., p\}$ and the memory recalls the output pattern $\mathbf{y}=\mathbf{y}^{\mu}$, then it is said that recall is correct.

The Alpha-Beta associative memories are based in order relations and binary operators [5]. For the learning stage the operator uses $\alpha$, and for the recall phase the operator uses $\beta$. These operators are defined as follows:

\begin{tabular}{|c|c|c|}
\hline$x$ & $y$ & $\alpha(x, y)$ \\
\hline 0 & 0 & 1 \\
\hline 0 & 1 & 0 \\
\hline 1 & 0 & 2 \\
\hline 1 & 1 & 1 \\
\hline
\end{tabular}

\begin{tabular}{|c|c|c|}
\hline$x$ & $y$ & $\beta(x, y)$ \\
\hline 0 & 0 & 0 \\
\hline 0 & 1 & 0 \\
\hline 1 & 0 & 1 \\
\hline 1 & 1 & 1 \\
\hline 2 & 0 & 2 \\
\hline 2 & 1 & 2 \\
\hline
\end{tabular}

Learning Phase. There are two kinds of Alpha-Beta associative memories, the Alpha-Beta associative memory MAX, denoted by $\mathbf{M}$ and the Alpha-Beta associative memory MIN, denoted by $\mathbf{W} . \wedge$ is used to denote minimum operator, and $\vee$ for the max operator. The ij-th entries of the associative memories $\mathbf{M}$ and $\mathbf{W}$ are defined by:

$$
m_{i j}=\bigvee_{\mu=1}^{p} \alpha\left(y_{i}^{\mu}, x_{j}^{\mu}\right) \quad w_{i j}=\bigwedge_{\mu=1}^{p} \alpha\left(y_{i}^{\mu}, x_{j}^{\mu}\right)
$$

respectively.

Recalling Phase. To obtain the output vector $\mathbf{y}$, the associative memory $\mathbf{M}$ or $\mathbf{W}$ will be operated with the input pattern $\tilde{\boldsymbol{x}}$. The i-th entry $y_{i}$ of the output pattern $\mathbf{y}$ is obtained as follows:

$$
y_{i}=\bigwedge_{j=1}^{n} \beta\left(m_{i j}, \tilde{x}_{j}\right) \quad y_{i}=\bigvee_{j=1}^{n} \beta\left(w_{i j}, \tilde{x}_{j}\right)
$$

for $\mathbf{M}$ and $\mathbf{W}$ respectively.

In either case $\mathbf{y}$ is called the recalled pattern. It is important to note that the associative memory $\mathbf{M}$ is robust to additive noise but sensitive to subtractive noise, and the associative memory $\mathbf{W}$ is robust to subtractive noise but sensitive to additive [5].

\section{Alpha Beta Associative Memory Committee}

In associative memories the learning phase carries the responsibility to obtain a generalization that achieves a good performance in recall phase. Enhancing this performance necesitates making multiple sub-generalizations per class. In 
order to generate these multiple sub-generalizations an Alpha-Beta Associative Memory Committee (ABAMC)is created. The ABAMC contains $m$ associative memories, each one specialized in one class. These sub-generalizations are made creating dynamically representative patterns using a similarity measure in the learning phase.

\subsection{Definitions}

Definition 1 (one-hot vector). A one hot vector is a vector $\mathbf{y}=\left[y_{1}, y_{2}, \ldots, y_{m}\right]$ with the characteristic that just one of its components is equal to one.

Definition 2 (Representative Pattern). Let $\mathbf{x}^{1}, \mathbf{x}^{2}, \ldots, \mathbf{x}^{l}$ be fundamental input patterns which are members of the same class $c$, where $l \in(1,2, \ldots, p)$. A representative pattern $\mathbf{r}=\left[r_{1}, r_{2}, \ldots, r_{n}\right]$ is an $n$ dimensional column vector with $\mathbf{r}=\mathbf{x}^{1} \cap \mathbf{x}^{2} \cap \ldots \cap \mathbf{x}^{l}$, where $\cap$ denotes intersection.

Definition 3 (Representative pattern identifier). Let $\boldsymbol{r}$ be a representative pattern, let $q \in[1, \ldots, p]$ be an integer value that indicates the number of representative patterns in a class. A representative pattern identifier is a one-hot column vector $\mathbf{v}=\left[v_{1}, v_{2}, . ., v_{q}\right]$ associated to $\mathbf{r}$ used to identify it.

Definition 4 (Operator $\zeta$ ). Let $\mathbf{x}=\left[x_{1}, x_{2}, \ldots, x_{n}\right]$ be an $n$ dimensional onehot vector. The operator $\zeta$ returns the unique position i, such that $x_{i}=1$, i.e.

$$
\zeta(\mathbf{x})=i \text { where } x_{i}=1
$$

Definition 5 ( Operator $\zeta^{-1}$ ). Let $a \in[1,2, . ., n]$ be an integer value. The operator $\zeta^{-1}$ is the inverse of $\zeta$, i.e.

$$
\zeta^{-1}(a)=\left[x_{1}, x_{2}, \ldots, x_{i}, \ldots, x_{n}\right] \text { where } x_{i}=\left\{\begin{array}{l}
0 i \neq a \\
1 i=a
\end{array}\right.
$$

Definition 6 (Alpha-Beta Max associative memory committee). Let $c \in[1,2, \ldots m]$ be an integer value that indicates a class in the fundamental set, let $\mathbf{r}^{c 1}, \mathbf{r}^{c 2}, \ldots, \mathbf{r}^{c q}$ be the $q$ representative patterns for the class $c$, let $\mathbf{v}^{c 1}, \mathbf{v}^{c 2}, \ldots, \mathbf{v}^{c q}$ be the pairwise distinguishing one-hot patterns, where $r^{\text {ck }}$ is associated to $\mathbf{v}^{\text {ck }}$ for $k \in[1,2, \ldots, q]$. An associative memory committee is the set of associative memories $\left\{\mathbf{M}^{c} \mid c\right.$ is a class\}, where $\mathbf{M}^{c}$ is the Max associative memory of the class $c$ that results in the learning of all associations $\left(\mathbf{r}^{c k}, \mathbf{v}^{c k}\right)$. The ij-th component $m_{i j}^{c}$ of $\mathbf{M}^{c}$ is defined as follows

$$
m_{i j}^{c}=\alpha\left(v_{i}^{c k}, r_{j}^{c k}\right)
$$


Definition 7 (Additive difference). Let $n$ be an integer value, $\boldsymbol{x}$ and $\boldsymbol{y}$ two patterns of dimension $n$. The additive difference is defined as the number of $y_{i}=1$ and $x_{i}=0$, and can be obtained using the following equation

$$
\delta(\mathbf{x}, \mathbf{y})=h((\mathbf{x} \cap \mathbf{y}), \mathbf{y})
$$

where $\cap$ denotes intersection and $h((\mathbf{x} \cap \mathbf{y}), \mathbf{y})$ is the hamming distance from intersection to pattern $\mathbf{y}$

Definition 8 (Representative pattern loss limit). Let $\boldsymbol{r}$ be a representative pattern, a representative pattern loss limit $a \in\{0,1,2, \ldots, n\}$ is a integer value that indicates the maximum number of $r_{i}=1$ that can change to $r_{i}=0$

Definition 9 (Input pattern loss limit). Let $\boldsymbol{x}$ be an input pattern, a input pattern loss limit $b \in\{0,1,2, \ldots, n\}$ is a integer value that indicates the maximum number of $x_{i}=1$ that can change to $x_{i}=0$

Definition 10 (operator $\psi$ ). Let $x \in\{0,1,2\}$ and $y \in\{0,1\}$. The operator $\psi$ is defined as follows

\begin{tabular}{|c|c|c|}
\hline$x$ & $y$ & $\psi(x, y)$ \\
\hline 0 & 0 & 1 \\
\hline 0 & 1 & 1 \\
\hline 1 & 0 & 0 \\
\hline 1 & 1 & 1 \\
\hline 2 & 0 & 0 \\
\hline 2 & 1 & 0 \\
\hline
\end{tabular}

Definition 11 (Operator $\eta$ ). Let $\mathbf{x}$ be a $n$ dimensional patter. Operator $\eta$ realizes a count of positions $x_{i}$ with $x_{i}=1$, is defined as follows:

$$
\eta=\sum_{i=1}^{n} x_{i}
$$

Definition 12 (Conditional similarity). Let $n$ be an integer value, $\mathbf{x}$ and $\mathbf{y}$ two patters of dimension $n$. The conditional similarity between $\mathbf{x}$ and $\mathbf{y}$ is defined as follows

$$
\varrho(\mathbf{x}, \mathbf{y})=\left\{\begin{array}{c}
\left(\frac{\eta(\mathbf{x})-h(\mathbf{x} \wedge \mathbf{y}, \mathbf{x})}{\eta(\mathbf{x})}+\frac{\eta(\mathbf{y})-h(\mathbf{x} \wedge \mathbf{y}, \mathbf{y})}{\eta(\mathbf{y})}\right) * \frac{1}{2} \text { if } \eta(\mathbf{x}) \neq 0, \eta(\mathbf{y}) \neq 0 \\
0 \quad \text { Otherwise }
\end{array}\right.
$$

\subsection{Learning Phase Algorithm}

The learning phase is shown with the following flow diagram. 


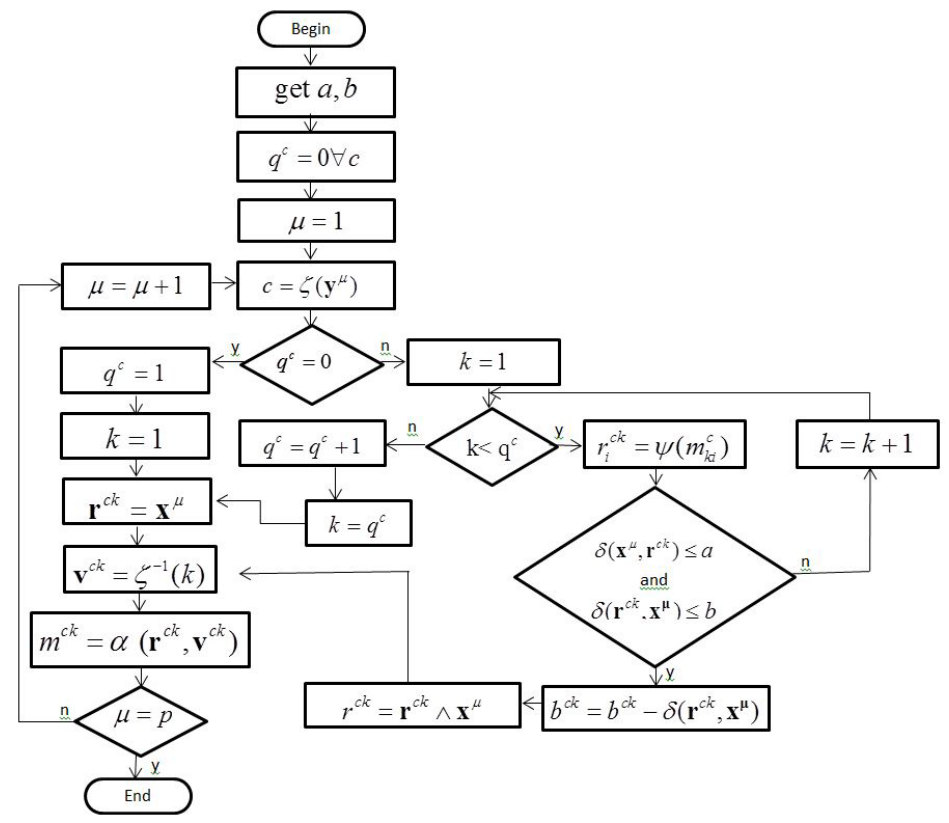

\subsection{Recalling Phase Algorithm}

The recalling phase is shown using the following flow diagram

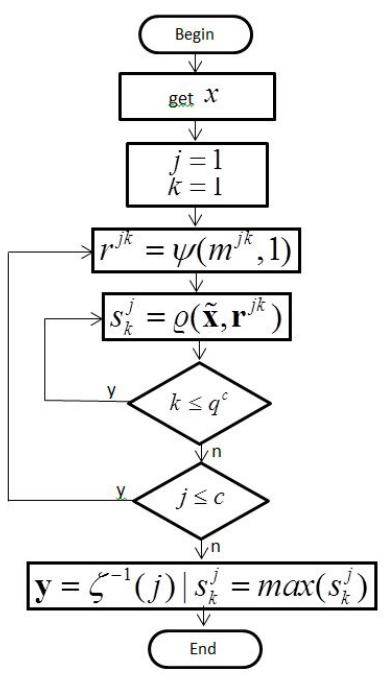

\section{Experimentation}

This section shows the result of experimentation made with the proposed algorithm. 
The algorithm was tested with three popular databases[10]: iris plant, wine and lung cancer, with every database we ran our algorithm several times with different loss values $a$ and $b$; these values and validation using 10 fold cross validation are shown. A comparison based on efficiency with other models in literature was made. Experiments with these models were realized using Weka[11/12. It is important to note the preprocessing realized to data before the experiments. This preprocessing consists of the following points:

1. Normalize numerical data to $[0,100]$

2. Convert this normalized data to Jonhson-Moebius code 13

3. Convert the class label into a One-Hot vector.

Experiments were made in a Intel core i5 computer with 4gb RAM, and are focused to show the efficiency with different learning schema, so two bayesian classifiers (BayesNet 14, NaiveBayes[15]), one function based classifier (Simple Logistic [16]), two meta-classifiers (AdaBoostM1 17, MultiClassClassifier [18]) and one decision tree classifier(RandomForest [19]) were used. The following table shows comparative efficiency between these models and ABAMC.

\begin{tabular}{|c|c|c|c|}
\hline & Iris Plant & Wine & Lung cancer \\
\hline BayesNet & 94.63 & 98.31 & 50 \\
\hline NaiveBayes & 94.63 & 94.94 & 62.5 \\
\hline Simple Logistic & 95.97 & 95.5 & 62.2 \\
\hline AdaBoostM1 & 95.3 & 88.2 & 46.87 \\
\hline MulticlassClassifier & 96.64 & 94.38 & 46.87 \\
\hline RandomForest & 94.63 & 97.75 & 56.25 \\
\hline ABAMC & $97.33(a=10, b=35)$ & $95.56(a=30, b=30)$ & $87.5(a=47, b=48)$ \\
\hline
\end{tabular}

The experimentation shows that the best efficiency with Iris plant and lung cancer databases was achieved by the proposed algorithm but was second in the wine database. This was anticipated in accordance with no free lunch theorem. The most promising result is with lung cancer database where ABAMC achieved 87.5 (25 percent more efficiency than other models).

\section{Conclusion and Future Work}

This model was conceived based on the hypothesis that the sub-generalizations obtained through representative patterns preserving useful information that is lost with only one generalization, and this preservation allows better recalling results. The experimentation confirms this hypothesis and provides motivation to test more challenging databases.

Future work would be in the following way:

1. It is highly desirable to find optimal ways to obtain the best loss value for an input pattern and for representative patterns.

2. Processes or modifications that enhance efficiency.

3. Complexity analysis for learning and recalling phase. 


\section{References}

1. Steinbuch, K., Piske, U.A.W.: Learning matrices and their applications. IEEE Transactions on Electronic Computers 12(6), 846-862 (1963)

2. Steinbuch, K.: Die lernmatrix. Kybernetik 1(3), 148-152 (1961)

3. Anderson, J.A.: A memory storage model utilizing spatial correlation. Kybernetik 5(3), 113-119 (1968)

4. Anderson, J.A.: A simple neural network generating an interactive memory. Mathematical Biosciences 14, 197-220 (1972)

5. Yáñez Márquez, C.: Associative memories based in binary operators and order relations. PhD thesis, Center for Computer Research (2002)

6. Yáñez Márquez, C., Diaz de Leon, J.L.: Associative memories based on order relations and binary operators. Computation and Systems 6(4), 300-311 (2003) (in Spanish)

7. Catalán-Salgado, E.A., Yáñez Márquez, C., José, A.C.A.: Simplification of the learning phase in the alpha-beta associative memories. In: CERMA 2008, pp. 428433 (2008)

8. Yáñez, C., Felipe-Riveron, E., López-Yáñez, I., Flores-Carapia, R.: A Novel Approach to Automatic Color Matching. In: Martínez-Trinidad, J.F., Carrasco Ochoa, J.A., Kittler, J. (eds.) CIARP 2006. LNCS, vol. 4225, pp. 529-538. Springer, Heidelberg (2006)

9. López-Yáñez, I., Yáñez Márquez, C., de la Sáenz-Morales, G.: Application of the gamma classifier to environmental data prediction. In: CERMA 2008, pp. 80-84. IEEE (2008)

10. Asuncion, A., Newman, D.: Uci machine learning repository (2007), http://archive.ics.uci.edu/ml/

11. Hall, M., Frank, E., Holmes, G., Pfahringer, B., Reutemann, P., Witten, I.H.: The weka data mining software: An update. SIGKDD Explorations 11(1), 10-18 (2009)

12. Hall, M., Frank, E., Holmes, G., Pfahringer, B., Reutemann, P., Witten, I.H.: Weka 3: Data mining software in java, http://www.cs.waikato.ac.nz/ml/weka/

13. Yáñez-Márquez, C., Sánchez-Fernández, L.P., López-Yáñez, I.: Alpha-Beta Associative Memories for Gray Level Patterns. In: Wang, J., Yi, Z., Żurada, J.M., Lu, B.-L., Yin, H. (eds.) ISNN 2006. LNCS, vol. 3971, pp. 818-823. Springer, Heidelberg (2006)

14. Christofides, N.: G.t.: An algorithmic approach. In: Press, A., (ed.): Computer Science and Applied Mathematics, Orlando, FL, USA (1975)

15. John, G.H., Langley, P.: Estimating continuous distributions in bayesian classifiers. In: Eleventh Conference on Uncertainty in Artificial Intelligence, pp. 338-345. Morgan Kaufmann, San Mateo (1995)

16. Sumner, M., Frank, E., Hall, M.: Speeding Up Logistic Model Tree Induction. In: Jorge, A.M., Torgo, L., Brazdil, P.B., Camacho, R., Gama, J. (eds.) PKDD 2005. LNCS (LNAI), vol. 3721, pp. 675-683. Springer, Heidelberg (2005)

17. Freund, Y., Schapire, R.E.: Experiments with a new boosting algorithm. In: Thirteenth International Conference on Machine Learning, San Francisco, pp. 148-156 (1996)

18. Witten, I.H., Frank, E.: Practical Machine Learning Tools, 2nd edn. Morgan Kaufmann Series in Data Management. Morgan Kaufmann Publishers Inc., USA (2005)

19. Breiman, L.: Random forests. Machine Learning 45(1), 5-32 (2001) 